\title{
Evaluation of rotary mode of application of ponies/horses for generation of electricity in Imphal, Manipur
}

\author{
R.K. TIWARI
}

\begin{abstract}
The animal driven rotary mode system was evaluated to generate electricity for battery charging. The cost economics of electricity generation and storage was worked out. The ponies energy in rotary unit to generate the electricity for battery charging was best utilized with proper work-rest cycle and nutritive feed. The main objective of the establishment of rotary power transmission system was to utilize power of pony during idle period. Fatigue score was found to be increased with duration of work. The physiological responses of the pony viz., pulse rate, respiration rate and rectal temperature increased with duration of work whereas speed of operation decreased. The battery (12 V, 35 Amp.-h) could be fully charged (specific gravity $=1.265$ ) after 6 hours of pony operation following work-rest cycle of half an hour: 1 hour rest. The CFL (8 W, DC) were used for test trial which were glowing brightly. The average speed of pony at no load and loads were 4 and 3 revolutions/min, respectively. The overall body length and girth of pony were 1150 and $1450 \mathrm{~mm}$, respectively. Time of charging was depended on the size of the battery as 35Ah battery required 6 hour for full charging. The alternator started emitting current at $1265 \mathrm{rpm}$ and at this stage the battery started drawing current. Voltage increased and current drawn decreased with the state of charging. The power output reduced with reduction in current drawn. Battery charging was recommended at 50 per cent discharge level. At specific gravity of 1.200 the battery is charged at 50 per cent level and the draught requirement was observed to be $53 \mathrm{kgf}$ which was well within the draught capacity of animals. Thus, it is recommended that battery should be put on charge at 50 per cent discharge level. Below this the draught requirement was observed high. The cost of pony and labour was therefore may be excluded from cost estimation. After excluding the cost of animal and labour the cost of battery charging was worked out as Rs. 9.62 and total cost for charging a battery of 35 Ah was found to be Rs. 57.72 .
\end{abstract}

KEY WORDS : Rotary mode, Battery fatigue work-rest, Rectal

How to cite this Article : Tiwari, R.K. (2015). Evaluation of rotary mode of application of ponies/horses for generation of electricity in Imphal, Manipur. Engg. \& Tech. in India, 6 (1) : 35-40. 